\title{
Noninvasive classification of liver disease in asymptomatic and oligosymptomatic male alcoholics
}

P. Borini ${ }^{1}$ and R.C. Guimarães ${ }^{2}$



Publication supported by FAPESP. $\ldots \ldots \ldots \ldots \ldots \ldots$

Received June 14, 2002 Accepted June 9, 2003
'Departamento de Clínica Médica, Faculdade de Medicina de Marília, Marília, SP, Brasil

${ }^{2}$ Departamento de Biologia Geral, Instituto de Ciências Biológicas, Universidade Federal de Minas Gerais, Belo Horizonte, MG, Brasil

\begin{abstract}
The predominant type of liver alteration in asymptomatic or oligosymptomatic chronic male alcoholics $(\mathrm{N}=169)$ admitted to a psychiatric hospital for detoxification was classified by two independent methods: liver palpation and multiple quadratic discriminant analysis (QDA), the latter applied to two parameters reported by the patient (duration of alcoholism and daily amount ingested) and to the data obtained from eight biochemical blood determinations (total bilirubin, alkaline phosphatase, glycemia, potassium, aspartate aminotransferase, albumin, globulin, and sodium). All 11 soft and sensitive, and 13 firm and sensitive livers formed fully concordant groups as determined by QDA. Among the 22 soft and not sensitive livers, 95\% were concordant by QDA grouping. Concordance rates were low (55\%) in the 73 firm and not sensitive livers, and intermediate (76\%) in the 50 not palpable livers. Prediction of the liver palpation characteristics by QDA was 95\% correct for the firm and not sensitive livers and moderate for the other groups. On a preliminary basis, the variables considered to be most informative by QDA were the two anamnestic data and bilirubin levels, followed by alkaline phosphatase, glycemia and potassium, and then by aspartate aminotransferase and albumin. We conclude that, when biopsies would be too costly or potentially injurious to the patients to varying extents, clinical data could be considered valid to guide patient care, at least in the three groups (soft, not sensitive; soft, sensitive; firm, sensitive livers) in which the two noninvasive procedures were highly concordant in the present study.
\end{abstract}

\section{Introduction}

Classification of types of lesion and identification of the occurrence of inflammatory activity in chronic alcoholics are important for understanding the natural history of alcoholism and for planning therapeutic strategies in an attempt to modify the evolution of
Key words

- Alcoholism

- Hepatopathy

- Noninvasive classification

- Liver palpation

- Laboratory exams

- Discriminant analysis 
histopathology, their differential diagnosis by noninvasive procedures is seriously impaired (8).

Nevertheless, the use of liver biopsies has several limitations in clinical practice. When indicated blindly, liver biopsies yielded on average $24 \%$ false-negative results (9), no evidence of necrosis or fibrosis was obtained for 40-50\% of chronic alcoholics (10), and the procedure was associated with significant morbidity and with $0.015-0.03 \%$ mortality in other series $(11,12)$. The costs of hospitalization and the discomfort imposed on the patients should also be considered. Asymptomatic or oligosymptomatic patients are not receptive to a liver biopsy and its indication is not ethically advisable. Therefore, efforts should be directed at characterizing the predominant type of liver lesion by noninvasive clinical and laboratory procedures.

More recently, a useful correlation has been found between histopathology and liver semiology $(3,13)$, serum biochemistry $(3,14-$ $16)$, ultrasound images $(17,18)$, and associations of clinical and biochemical data $(13,19)$. Thus, it has been demonstrated that isolated or combined noninvasive methods can be of help in identifying hepatic damage and, to a certain extent, in evaluating the type, extent and severity of tissue damage (20).

The present study was designed to determine the significance of the simple classification of liver state by palpation in asymptomatic or oligosymptomatic chronic alcoholics admitted to a psychiatric ward for alcohol detoxification. Independent classification was attempted using a variety of statistical procedures applied to patient data obtained by anamnestic and physical examination by an experienced clinician and to a set of blood biochemical determinations. Data of interest to the clinician were collected using a standard structured protocol and the statistical team screened them to select the variables that were found to be useful and adequate for the tests. Concordance between the two ap- proaches could increase confidence in the presumptive clinical diagnosis of liver disease, and definition of more important quantitative variables would start a process of improvement of statistical classification which would be of help in terms of patient care.

\section{Material and Methods}

Patients were 169 asymptomatic or oligosymptomatic male alcoholics with no other clinical problems admitted to a psychiatric hospital for detoxification, for whom complete data were available. Hospital admission was indicated by psychiatrists after clinical examination at ambulatory services of the Marília Medical School. No patient had used illicit drugs or other medicines during the preceding 30 days.

A structured protocol for clinical and physical examination, developed by one of the authors (P.B.) (21), was applied by him during the first $24 \mathrm{~h}$ of hospitalization. No patient showed neurological signs compatible with liver insufficiency or clear symptoms of liver dysfunction.

Hepatomegaly was diagnosed with the patient in the supine position when the liver was palpable more than $1.5 \mathrm{~cm}$ below the costal margin along the right hemiclavicular line, with the upper level of liver percussion not being lower than the fifth intercostal space (22).

The patients were divided into five groups on the basis of the liver being not palpable or enlarged, with the latter condition involving the following four groups according to the firmness observed and the patient's report of pain at palpation (Table 1): soft, not sensitive; firm, not sensitive; soft, sensitive; firm, sensitive.

Blood counts, feces parasitology and blood biochemical determinations were conducted during the first $48 \mathrm{~h}$ of hospitalization after the patient gave informed consent. The protocol was approved by the Hospital Eth- 
ics Committee. Patients were free from Schistosoma mansoni eggs in the feces. Viral antigens were not searched for.

All qualitative variables (18 clinical and 2 from laboratory exams) were excluded from the statistical analysis. Among 18 quantitative variables, spleen palpation (in $\mathrm{cm}$ below the costal margin) was excluded since it was not obtained in most cases. The liver palpation data (in cm below the costal margin; mean $\pm \mathrm{SD}$ ), obtained for 169 patients, were subjected only to descriptive analysis of variance: soft, not sensitive $(\mathrm{N}=22,5.07$ $\pm 2.07)$; firm, not sensitive ( $\mathrm{N}=73,6.28 \pm$ 2.37); soft, sensitive $(\mathrm{N}=11,5.67 \pm 2.87)$; firm, sensitive $(\mathrm{N}=13,6.14 \pm 1.70)$, and not palpable $(\mathrm{N}=50)$.

Results for cases with complete data regarding the 16 selected variables were subjected to multivariate analysis of variance (MANOVA) only for exploratory purposes due to the fact that the data did not satisfy the requirement for equality of the matrices of variance and covariance among groups. The descriptive level found was 0.091, higher than the 5\% acceptance level for the group effect. Multiple quadratic discriminant analysis (QDA; MINITAB (1987), version 6.1.1, Minitab Inc., State College, PA, USA) was applied for classification since this analysis, unlike its linear version, is free from the requirement for equality of matrices. Nevertheless, six additional variables were excluded as the statistician's decision, without consultation with the clinician, due to the required avoidance of biases. Among the highly intercorrelated variables, only one of each set was retained: of the three measurements of bilirubin, only the total, and of the two aminotransferases, only the aspartate one. The three variables concerning complex ions were excluded altogether because they were intercorrelated and also correlated with other ones, specifically in the soft, sensitive group. Variables applicable to QDA were then only two obtained from anamnesis (years of alcoholism, grams of alcohol con- sumed per day) and eight obtained from blood laboratory data: total albumin, globulin and bilirubin levels, and aspartate aminotransferase, alkaline phosphatase, fasting glucose, sodium and potassium levels.

Cluster analysis, which works well for other types of variables in liver and brain histopathology (23), was applied using the complete linkage (farthest neighbors) protocol. However, since this protocol did not provide interesting results for the present purposes, it will not be detailed. A preliminary account of the statistical treatment of the full data is available in the Cordani and Gori report (24).

\section{Results}

Skin color distribution according to common criteria for the Brazilian population was $72 \%$ white, $15 \%$ black and $13 \%$ intermediate. The majority of patients had low income and little schooling. Eighty percent were tobacco smokers and average age was 39 years (range 20 to 79), with no significant differences among groups. The mean values of the variables studied are presented in Table 1.

Classification by QDA produced exactly five 'statistical' groups which could be compared to the five liver palpation ('clinical') groups (Table 2). Overall concordance between clinical and statistical groups (number of concordant cases/total cases) was $73 \%$. Cohen's kappa coefficient (25) applied to the matrix (Table 2) was 0.64; this level of agreement between the two procedures may be informally evaluated as substantial to moderate according to the Landis and Koch (26) 'benchmark' classes.

Comparisons through rows indicated full concordance of statistical groups with clinical groups for soft, sensitive and firm, sensitive and $95 \%$ concordance for soft, not sensitive cases. The firm, not sensitive and not palpable groups corresponded to worse statistical classification, overlapped widely with 
one another and were also the most numerous, although it may be suggested that the not palpable group was more homogeneous than the firm, not sensitive group. Comparisons through columns, indicating the positive predictive value of the statistical procedure, ran in the opposite order of the levels of concordance read through rows, reaching $95 \%$ for the firm, not sensitive group and lower values for the other groups.

The patterns formed by QDA could be better interpreted on the basis of the ratios between group means and the overall mean for each variable. The range of these ratios is shown in Table 1, indicating the informative value or 'weight' of a variable, albeit roughly and as a first approach. Intergroup differences were greater $(>20 \%)$ for the patient's information about the duration of the habit and the daily amount drunk, and for total bilirubinemia. Highest daily consumption characterized painful ('inflammatory') livers, with firmness developing with longer duration of the habit. Lower daily consumption was reported by patients with firm, not sensitive ('cirrhotic') livers, and intermediate levels by patients with soft, not sensitive ('degenerative') livers. Intermediate inter-

Table 1. Anamnestic and blood biochemistry data utilized for multiple quadratic discriminant analysis in the liver palpation groups of asymptomatic or oligosymptomatic chronic alcoholics.

\begin{tabular}{lccccccc}
\hline & $\begin{array}{c}\text { Soft, not } \\
\text { sensitive }\end{array}$ & $\begin{array}{c}\text { Soft, } \\
\text { sensitive }\end{array}$ & $\begin{array}{c}\text { Firm, } \\
\text { sensitive }\end{array}$ & $\begin{array}{c}\text { Firm, not } \\
\text { sensitive }\end{array}$ & $\begin{array}{c}\text { Not } \\
\text { palpable }\end{array}$ & $\begin{array}{c}\text { Total } \\
\text { Range } \\
\text { of ratios }\end{array}$ \\
\hline Years of habit & $19.2 \pm 7.8$ & $16.2 \pm 6.3$ & $22.1 \pm 12.7$ & $24.5 \pm 11.4$ & $21.1 \pm 10$ & $22.0 \pm 10.6$ & 0.37 \\
Alcohol (g/day) & $316 \pm 230$ & $389 \pm 202$ & $357 \pm 175$ & $296 \pm 186$ & $302 \pm 228$ & $316 \pm 205$ & 0.29 \\
Bilirubin (mg/dl) & $0.94 \pm 0.13$ & $0.95 \pm 0.40$ & $1.14 \pm 0.60$ & $1.06 \pm 0.54$ & $0.93 \pm 0.17$ & $1.00 \pm 0.41$ & 0.21 \\
AP (IU) & $41.2 \pm 17.1$ & $46.5 \pm 17.9$ & $43.5 \pm 24.3$ & $47.2 \pm 20.6$ & $44.0 \pm 20.6$ & $45.1 \pm 20.2$ & 0.14 \\
Glycemia (mg/dl) & $76.4 \pm 11.9$ & $69.5 \pm 12.8$ & $80.6 \pm 19.1$ & $77.3 \pm 20.2$ & $74.3 \pm 17.5$ & $75.9 \pm 18.0$ & 0.14 \\
Potassium (mEg/l) & $4.39 \pm 0.65$ & $4.26 \pm 0.48$ & $3.85 \pm 0.77$ & $4.18 \pm 0.72$ & $4.35 \pm 0.64$ & $4.24 \pm 0.68$ & 0.13 \\
AST (IU) & $15.3 \pm 7.2$ & $14.3 \pm 6.2$ & $13.9 \pm 4.6$ & $15.1 \pm 9.3$ & $14.4 \pm 6.3$ & $14.8 \pm 7.7$ & 0.09 \\
Albumin (g/dl) & $3.57 \pm 0.48$ & $3.73 \pm 0.69$ & $3.56 \pm 0.52$ & $3.49 \pm 0.65$ & $3.67 \pm 0.60$ & $3.58 \pm 0.61$ & 0.07 \\
Globulin (g/dl) & $3.56 \pm 0.67$ & $3.66 \pm 0.68$ & $3.59 \pm 0.79$ & $3.68 \pm 1.0$ & $3.56 \pm 0.78$ & $3.62 \pm 0.87$ & 0.04 \\
Sodium (mEq/l) & $139.1 \pm 4.52$ & $140.9 \pm 4.36$ & $139.9 \pm 3.88$ & $139 \pm 1.0$ & $139.7 \pm 4.59$ & $139 \pm 4.7$ & 0.01 \\
\hline
\end{tabular}

$\mathrm{AP}=$ alkaline phosphatase $; \mathrm{AST}=$ aspartate aminotransferase. Data are reported as means $\pm \mathrm{SD}$. Range of ratios $=$ higher-lower.

Table 2. Classification of liver disease in asymptomatic or oligosymptomatic alcoholics by liver palpation and multiple quadratic discriminant analysis (QDA) applied to some quantitative variables.

\begin{tabular}{|c|c|c|c|c|c|c|c|}
\hline \multirow[t]{2}{*}{ Liver palpation } & \multicolumn{5}{|c|}{ ODA } & \multirow[t]{2}{*}{ Total } & \multirow[t]{2}{*}{$\%$ concordant } \\
\hline & I & II & III & IV & V & & \\
\hline Soft, not sensitive & 21 & 1 & & & & 22 & 95 \\
\hline Soft, sensitive & & 11 & & & & 11 & 100 \\
\hline Firm, sensitive & & & 13 & & & 13 & 100 \\
\hline Firm, not sensitive & 6 & 4 & 8 & 40 & 15 & 73 & 55 \\
\hline Not palpable & 7 & 2 & 1 & 2 & 38 & 50 & 76 \\
\hline Total & 34 & 18 & 22 & 42 & 53 & 169 & 85 \\
\hline$\%$ concordant & 62 & 61 & 59 & 95 & 72 & 70 & 73 \\
\hline
\end{tabular}


group differences (13-14\%) were observed for alkaline phosphatase, glucose and potassium levels, while the differences for aspartate aminotransferase and albumin were already below $10 \%$ and those for globulins and sodium below $5 \%$.

\section{Discussion}

Hepatomegaly was present in $70 \%$ of our sample, close to the $80 \%$ value reported for active alcoholics (27). The correlation between the histopathologic alterations and liver palpation characteristics may indicate that in the presence of predominantly degenerative changes (hydropic or fatty alterations) the liver became softer but remained insensitive, while in the presence of inflammation, associated or not with degeneration, it became sensitive. Progressive hardening followed accumulation of fibrotic tissue $(28,29)$. Among the clinical signs, a firm liver showed the highest accuracy for the diagnosis of cirrhosis $(13,30)$. Liver enlargement was well correlated with alcoholism duration and histopathologic findings (31). Other clinical studies on alcoholism patterns similar to the present one have further clarified some significant differences in blood biochemical tests according to the presence of hepatomegaly $(19,32)$ and to the characteristics of firmness or painfulness of the liver (19).

Nosologic diagnosis is a multivariate compositional procedure relying mostly on the clinician's experience in aggregate pattern formation. Discriminant analysis is a sophisticated but commonly utilized statistical technique, which was found to be useful here for classification based on signs, symptoms and a variety of simple biochemical determinations. When palpation characteristics were considered as classification standards, the use of only two anamnestic variables and eight biochemical values yielded $73 \%$ concordant cases. Most of the discordance resided in the hard and not sensitive and in the nonpalpable livers, which might be consid- ered together as cases of mostly chronic or long-lasting lesions, while most of the concordance $(\geq 95 \%)$ coincided with cases of acute degenerative and/or inflammatory hepatomegalic damage.

Dispersion observed in the hard and not sensitive and in the nonpalpable livers demonstrated the occurrence of confounding factors amongst themselves and between them and other groups. The former showed the highest statistical dispersion (55\% concordance), indicative of cirrhosis with varying intensity of superimposed degenerative or inflammatory processes. Otherwise, the statistical prediction of cases belonging to this group was satisfactory (95\%). The latter was somewhat more homogeneous and $76 \%$ concordant, indicative of predominance of cases with extreme fibrosis leading to reduction of liver dimensions but also possibly involving cases of originally small livers and alterations insufficient to determine hepatomegaly.

Since among all the effects of alcohol on liver only cirrhosis is irreversible with abstinence and since $13-30 \%$ of alcoholics become cirrhotic (33), about $60 \%$ of cirrhosis cases manifest through complications (34) and about $50 \%$ of cases of alcoholic hepatitis without fibrotic superimposition will develop cirrhosis during resolution of the inflammatory process (35), it becomes evident that an early diagnosis of the type of lesion is essential. The greatest benefit would be achieved for patients with potentially reversible degenerative or inflammatory lesions by the implementation of abstinence plus antiinflammatory $(2,14,35)$ and anti-fibrosis (36) therapies.

Using two independent approaches, the present study indicated that a noninvasive presumptive diagnosis of certain types of liver injury can be made by simple and inexpensive procedures. Fortunately, the highest accuracy of the clinical and statistical classifications coincided with the groups whose prognosis would be better with the institution of adequate therapy. It is not our inten- 
tion to suggest that the 'gold standards' of histopathologic data can be disregarded. What we wish to emphasize is that, when these procedures are too costly or potentially injurious to patients to varying extents, careful and reliable clinical data should be con- sidered adequate for the planning of care.

\section{Acknowledgments}

We wish to thank Carlos Henrique Costa Moreira for final statistical assistance.

\section{References}

1. Maddrey WC (1998). Alcoholic hepatitis: clinicopathologic features and therapy. Seminars in Liver Disease, 8: 91-102.

2. Ramond MJ, Poynard T, Rueff B, Mathurin P, Theodore C, Chaput JC \& Benhamou JP (1992). Randomized trial of prednisolone in patients with severe alcoholic hepatitis. New England Journal of Medicine, 326: 507-512.

3. Sherlock S \& Dooley J (1993). Assessment of liver function. In: Sherlock C \& Dooley J (Editors), Disease of the Liver and Biliary System. 9th edn. Blackwell Scientific Publications, London, UK, 1732.

4. Fleming KA \& McGee JO'D (1984). Alcohol induced liver disease. Journal of Clinical Pathology, 37: 721-733.

5. Brunt PW, Kew MC, Scheurer PT \& Sherlock S (1974). Studies in alcoholic liver disease in Britain. Gut, 15: 52-58.

6. Insunza I, Iturriaga $H$, Ugarte G \& Altschiller H (1971). Clinical and histological liver abnormalities in alcoholics. Acta HepatoSplenologica, 18: 460-470

7. Frederiks WM, Myagkaya GL, Bosch KS, Fronik GM, van Veen $\mathrm{H}_{\text {, }}$ Vogels IM \& James J (1983). The value of enzyme leakage in the prediction of necrosis in liver ischemia. Histochemistry, 78: 459-472.

8. Rankin JGD, Orrego-Matte H, Deschenes J, Medline A, Findlay JE \& Armstrong AIM (1978). Alcoholic liver disease: the problem of diagnosis. Alcoholism, Clinical and Experimental Research, 2: 327-338.

9. Nord JH (1982). Biopsy diagnosis of cirrhosis: blind percutaneous versus guided direct vision techniques. A review. Gastrointestinal Endoscopy, 28: 102-104

10. Diel AM (1989). Doença hepática alcoólica. Clínicas Médicas da América do Norte, 4: 899-917.

11. Piccinino F, Sagnelli E, Pasquale G \& Guisti G (1986). Complications following percutaneous liver biopsy: a multicentre retrospective study on 68,276 biopsies. Journal of Hepatology, 2: 165-173.

12. Schalm SW (1997). The diagnosis of cirrhosis: clinical relevance and methodology. Journal of Hepatology, 27: 1118-1119.

13. Oberti F, Valsesia E, Pilette C, Rousselet MC, Bedossa P, Aubé C, Gallois Y, Rifflet H \& Maiga MY (1997). Noninvasive diagnosis of hepatic fibrosis or cirrhosis. Gastroenterology, 113: 1609-1616.

14. Gupta S, Slaughter S, Akriviadis EA, Valenzuela R \& Deodhar SD (1995). Serial measurement of serum C-reactive protein facilitates evaluation in alcoholic hepatitis. Hepato-Gastroenterology, 42: 516521.

15. Poynard T, Aubert A, Bedossa P, Abella A, Naveau S, Paraf F, Chaput JC \& Benhamou JP (1991). A simple biological index for detection of alcoholic liver disease in drinkers. Gastroenterology, 100: 1397-1402.

16. Teare JP, Sherman D, Greenfield SM, Simpson J, Bray G, Catterall AP, Murray-Lion IM, Peters TJ, Williams R \& Thompson RP (1993). Comparison of serum procollagen III peptide concentrations and PGA index for assessment of hepatic fibrosis. Lancet, 342: 895-898.

17. Di Lelio A, Cestari C, Lomazzi A \& Beretta L (1989). Cirrhosis: diagnosis with sonographic study of the liver surface. Radiology, 172: 389-392.

18. Sumino Y, Kravetz D, Kanel GC, McHutchison JG \& Reynolds TB (1993). Ultrasonographic diagnosis of acute alcoholic hepatitis 'pseudoparallel channel sign' of intrahepatic artery dilatation. Gastroenterology, 105: 1477-1482.

19. Borini $P$ (1991). Avaliação do envolvimento hepático em alcoolistas crônicos assintomáticos ou oligossintomáticos. 2 - Aspectos laboratoriais. Gastroenterologia Endoscopia Digestiva, 10: 137-143.

20. Malaguarnera M, Giugno I, Trovato BA, Panebianco MP, Restuccia N \& Ruello P (1996). Lipoprotein(a) in cirrhosis. A new index of liver functions? Current Medical Research and Opinion, 14: 479-485.

21. Borini P (1990). Anamnese psiquiátrica e clínica, exame físico e exames complementares estruturados e informatizados para aplicação em alcoolistas. Jornal Brasileiro de Psiquiatria, 39: 250-265.

22. Porto JAF (1987). Aparelho digestivo. In: Tarantino AB (Coordenador), Vieira Romeiro Semiologia Médica. Vol. 2. 12th edn. Guanabara Koogan, Rio de Janeiro, RJ, Brazil, 343-446.

23. Brasileiro-Filho G, Guimarães RC \& Pittella JEH (1989). Quantification of cerebral neuroglia and endothelial cells, and karyometry of neuroglia in liver cirrhosis and schistosomiasis mansoni. Acta Neuropathologica, 77: 582-590.

24. Cordani LK \& Gori MEG (1992). Agrupamento de pacientes alcoolistas a partir de exames clínicos e laboratoriais. Relatório de análise estatística no 9207, do Centro de Estatística Aplicada, Instituto de Matemática e Estatística, Universidade de São Paulo, São Paulo, $\mathrm{SP}$, Brazil.

25. Cohen J (1960). A coefficient of agreement for nominal scales. Educational and Psychological Measurement, 20: 37-46.

26. Landis JR \& Koch GC (1977). The measurement of observer agreement for categorical data. Biometrics, 33: 159-174.

27. Mincis M (1997). Doença hepática alcoólica. In: Mincis M (Editor), Gastroenterologia e Hepatologia. Lemos-Editorial, São Paulo, SP, Brazil, 669-690.

28. Bates B (1990). Propedêutica Médica. 4th edn. Editora Guanabara, Rio de Janeiro, RJ, Brazil, 337.

29. Porto CC (1987). Exame Clínico. Guanabara, Rio de Janeiro, RJ, Brazil, 312-341.

30. Tiné F, Catalgirone M, Camma C et al. (1990). Clinical indicators of compensated cirrhosis: a prospective study. In: Dianzani UM \& Gentilini P (Editors), Chronic Liver Damage. Elsevier, Amsterdam, The Netherlands, 187-198.

31. Ugarte G, Iturriaga H \& Insuriza I (1970). Some effects of ethanol on normal and pathologic livers. In: Popper H \& Schaffner F (Editors), Progress in Liver Disease. Vol. 3. Grune \& Stratton, New York, 355.

32. Neves MM, Ambroini Jr O, Kondo M, Ferrari Jr AP, Oliveira LCM \& Borges DR (1986). Clinical-laboratory evaluation of the liver in alcohol addicts. Preliminary results. Gastroenterologia Endoscopia Digestiva, 5: 77-80 
33. Popper $\mathrm{H}$ (1977). The pathogenesis of alcoholic cirrhosis. In: Fisher MM \& Rankin JG (Editors), Alcohol and the Liver. Plenum Press, New York, 289-307.

34. Saunders JB, Walters JRF, Davis P \& Paton A (1981). A 20-year prospective study of cirrhosis. British Medical Journal, 282: 263266.
35. Helman RA, Temko MH, Nye SW \& Fallon HJ (1971). Alcoholic hepatitis. Natural history and evaluation of prednisolone therapy. Annals of Internal Medicine, 74: 311-321.

36. Kershenobich D, Vargas F, Garcia-Tsao G, Pérez-Tamayo R, Gent M \& Rojkind M (1988). Colchicine in the treatment of cirrhosis of the liver. New England Journal of Medicine, 318: 1709-1713. 